\title{
Postoperative infections are associated with adverse outcome after resection with curative intent for colorectal cancer
}

\author{
HIRONORI TSUJIMOTO, HIDEKI UENO, YOJIRO HASHIGUCHI, \\ SATOSHI ONO, TAKASHI ICHIKURA and KAZUO HASE \\ Department of Surgery, National Defense Medical College, \\ Tokorozawa 359-8513, Japan
}

Received May 7, 2009; Accepted August 7, 2009

DOI: $10.3892 /$ ol_00000022

\begin{abstract}
This study focused on the impact of post-operative infection on patient outcome after resection with curative intent for colorectal cancer. Postoperative surgical and medical complications have been implicated as a negative predictor of long-term outcome in various malignancies. We studied a population of 1083 patients who underwent resection with curative intent for colorectal cancer. These patients were divided into 2 groups based on the occurrence (65 patients, $6 \%$ ) or absence (1018 patients, 94\%) of postoperative complications due to infection. We investigated the demographic and clinicopathological features of each patient with and without postoperative infectious complications, as well as the impact of postoperative infection on long-term survival. Results showed that patients with postoperative infectious complications had diabetes mellitus more frequently and also had urgent surgery compared to those without infectious complications. In addition, patients with postoperative infectious complications had a significantly more unfavorable outcome compared with those without postoperative infection in cancer-specific, but not overall survival. Multivariate analysis demonstrated that age, rectal cancer and tumor stage correlated with overall survival, but not postoperative infectious complications. However, postoperative infections, as well as gender, were associated with the length of time until the patient succumbed from the recurrence of colorectal cancer after resection for curative intent. Thus, postoperative infectious complications are predictors of adverse clinical outcome in patients with colorectal cancer. However, further immunological study is necessary to confirm the biological significance of these findings.
\end{abstract}

Correspondence to: Dr Hironori Tsujimoto, Department of Surgery, National Defense Medical College, 3-2 Namiki, Tokorozawa 359-8513, Japan

E-mail: tsujihi@ndmc.ac.jp

Key words: colorectal cancer, postoperative infectious complications, prognosis, recurrence

\section{Introduction}

Considerable advances have been made in the areas of prevention, diagnosis and therapy. However, postoperative infections following major surgery for gastro-intestinal cancer are the most frequent complications that are noted. Complications due to postoperative infection result in an increased cost of treatment, longer hospital stay and ultimately, premature mortality $(1,2)$.

Many researchers have reported that postoperative surgical and medical complications may contribute to a high rate of recurrence and unfavorable long-term survival in various malignancies such as cervical, colorectal and esophageal cancer (3-5). Increasing evidence has shown that postoperative complications due to infection, especially those caused by anastomotic leakage after colorectal surgery, were significantly associated with a negative long-term outcome $(3,6-8)$. In contrast, beneficial effects of postoperative emphysema and intrapleural infection have been reported after pneumonectomy for lung cancer. Thus, whether or not infection and/or febrile complication after surgery is associated with the long-term survival of patients after curative resection for various types of cancer is still controversial (8-11).

This study focused on the impact of postoperative infection on long-term survival following resection with curative intent for colorectal cancer.

\section{Materials and methods}

Patients. Between January 2002 and December 2007, 1669 consecutive patients underwent colorectal surgery at the National Defense Medical College Hospital (Tokorozawa, Saitama, Japan). Among the 1669 patients, 1083 patients (632 men, 451 women; mean age $64.5 \pm 11.0$ years, range $18-96$ years) who underwent colorectal surgery with resection with curative intent were enrolled in this study. The population of 1083 patients was then divided into 2 groups based on the occurrence (65 patients, $6 \%$ ) or absence (1018 patients, 94\%) of complications due to postoperative infection. If no gross residual disease was evident at the time of the operation and the margins of resection were tumor-free on histological examination, the surgery was considered to be curative. Resected specimens were examined histopathologically and were staged according to the International Union Against Cancer (UICC) 
TNM classification of malignant tumors (12). Adjuvant therapy by oral anti-cancer agents such as 5-fluorouracil (UFT) was recommended for patients with disease stage III or IV, or those with a high potential of recurrence based on the pathological findings. Adjuvant therapy was then performed in 31 (47.7\%) patients with postoperative infectious complications and $466(45.8 \%)$ patients without infectious complication. These patients were retrospectively evaluated for their pre- and postoperative status, pathological findings and surgical procedure, according to our computer database or medical and nursing charts.

Definition of infectious complications. Complications due to postoperative infection were defined by a combination of clinical findings and the results of both laboratory and other tests recorded in medical records. Clinical evidence was derived from direct observation of the infection site or from reviewing patient charts. Laboratory evidence included culture results, antigen or antibody detection tests, or analysis by microscopic visualization. Supportive data were derived from other diagnostic studies, such as X-ray, ultrasonography (US) and computed tomography (CT). In our study, complications from postoperative infection included anastomotic leakage (identified by radiography or clinical suspicion), urinary tract infection (pyrexia with microbiological evidence), bacteremia (pyrexia with microbiological evidence), intraperitoneal abscess (pyrexia with fluid collection diagnosed by US or CT, or identified radiographically), pneumonia (pyrexia with infiltrate on chest X-ray), pseudomembrane colitis (identified by colonoscopy and microbiological evidence) and central venous catheter-related infections (pyrexia with microbiological evidence). This study included a wound infection involving deep soft tissues, e.g., facial and muscle layers, but excluded infection involving only skin and subcutaneous tissue of the skin because of its minimal effect on the systemic immune response.

Follow-up. Survival time was measured from the date of resection to the date the patient succumbed due to any cause. Patients who survived were censored in our survival analysis. The patients were observed at our hospital or the outpatient clinic at 3- to 4-month intervals during the first 2 years of the study, and then every 6 or 12 months for 3 years. After 5 years, an annual follow-up was conducted through telephone conversations with the patients, their family or practitioner.
Table I. Frequency of complications due to postoperative infection.

\begin{tabular}{lrc}
\hline Infectious complications & No. & $\%$ \\
\hline Anastomotic leakage & 29 & 2.7 \\
Wound infection & 25 & 2.3 \\
Urinary tract infection & 5 & 0.5 \\
Bacteremia & 3 & 0.3 \\
Intraperitoneal abscess & 2 & 0.2 \\
Pneumonia & 2 & 0.2 \\
Pseudomembrane colitis & 1 & 0.1 \\
Central venous catheter- & 1 & 0.1
\end{tabular}

related infections

Total

68

Definition of recurrence patterns. Recurrences were identified by CT, positron emission tomography (PET), US or colonoscopy. They were classified as locoregional, liver, lung, brain, bone, distant lymph node and peritoneal recurrence. Recurrence at cervical, celiac or paraaortic lymph nodes was classified as distant lymph node recurrence. When simultaneous recurrences were detected, the site of massive and/or life-threatening recurrence was regarded as a main pattern of recurrence.

Statistical analysis. Statistical calculations were performed using StatView version 5.0 (SAS Institute, Inc., Cary, NC, USA). Data are expressed as mean \pm SD. Statistical analyses were performed using either the Mann-Whitney U or Chisquare tests with Fisher's exact test, as appropriate. Survival rates were obtained using Kaplan-Meier, and the significance of the difference in the survival rate was determined by a log-rank test. Univariate and multivariate analyses were performed using the Cox proportional hazards model. $\mathrm{P}<0.05$ was considered statistically significant.

\section{Results}

Frequencies of complications due to postoperative infection are shown in Table I. Anastomotic leakage was the most frequent infectious complication after colorectal cancer, followed by wound infection. No difference was noted in the age, gender and incidence of hypertension between patients with

\section{Overall survival}

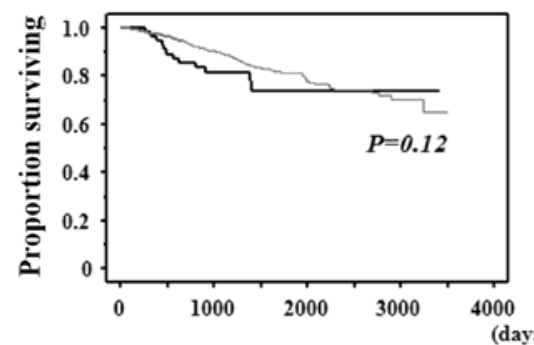

Cancer-specific survival

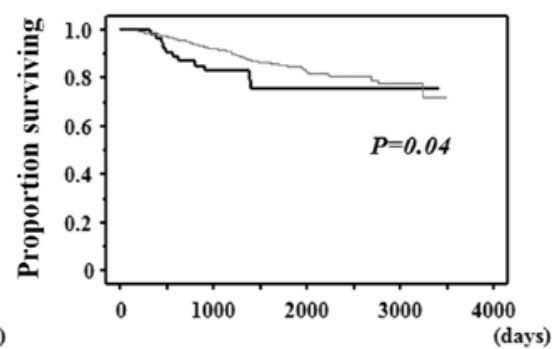

Figure 1. Overall and cancer-specific survival after surgery with curative intent for colorectal cancer. Bold and fine lines indicate survival curves for patients with and without postoperative infectious complications, respectively. P-values of the log-rank test are shown in each graph. 
Table II. Demographic and clinicopathological data of patients with or without postoperative complications due to infection.

\begin{tabular}{|c|c|c|c|}
\hline & Complications & No complications & P-value \\
\hline Number & 65 & 1018 & \\
\hline Age & $65.6 \pm 9.0$ & $64.5 \pm 11.1$ & 0.44 \\
\hline Gender (M:F) & $44: 21$ & $588: 430$ & 0.11 \\
\hline \multicolumn{4}{|l|}{ Co-morbidity } \\
\hline \multicolumn{4}{|l|}{ Hypertension } \\
\hline Yes & $10(15.4)$ & $159(15.6)$ & 0.99 \\
\hline No & $50(84.6)$ & $859(84.4)$ & \\
\hline \multicolumn{4}{|l|}{ Diabetes mellitus } \\
\hline Yes & $13(20.0)$ & $86(8.5)$ & 0.01 \\
\hline No & $52(80.0)$ & $932(91.5)$ & \\
\hline \multicolumn{4}{|l|}{ Emergency surgery } \\
\hline Yes & $2(3.1)$ & $4(0.4)$ & 0.04 \\
\hline No & $63(96.9)$ & $1014(99.6)$ & \\
\hline \multicolumn{4}{|l|}{ Location } \\
\hline Colon & $30(46.2)$ & $532(52.3)$ & 0.31 \\
\hline Rectum & $35(53.8)$ & $474(57.7)$ & \\
\hline \multicolumn{4}{|l|}{ Laparoscopic surgery } \\
\hline Yes & $3(4.6)$ & $30 \quad(2.9)$ & 0.44 \\
\hline No & $62(95.4)$ & $988(97.1)$ & \\
\hline \multicolumn{4}{|l|}{ Inflammatory bowel disease } \\
\hline Yes & $0(0.0)$ & $2(0.2)$ & 0.99 \\
\hline No & $65(100)$ & $1016(99.8)$ & \\
\hline \multicolumn{4}{|l|}{ Stoma construction } \\
\hline Yes & $16(24.6)$ & $159(15.6)$ & 0.07 \\
\hline No & $49(75.4)$ & $859(84.4)$ & \\
\hline \multicolumn{4}{|l|}{ Curability } \\
\hline A & $6 \quad(9.2)$ & $51 \quad(5.0)$ & 0.15 \\
\hline B & $59(90.8)$ & $967(95.0)$ & \\
\hline \multicolumn{4}{|l|}{ Stage } \\
\hline 0 & $1 \quad(1.5)$ & $33(3.2)$ & 0.50 \\
\hline I & $14(21.5)$ & $222(21.8)$ & \\
\hline II & $23(35.4)$ & $336(33.0)$ & \\
\hline III & $22(33.8)$ & $388(38.1)$ & \\
\hline IV & $5 \quad(7.7)$ & $39(3.8)$ & \\
\hline \multicolumn{4}{|l|}{ Outcome } \\
\hline Alive & $49(75.4)$ & $826(81.1)$ & 0.28 \\
\hline Succumbed to tumor relapse & $12(18.5)$ & $122(12.0)$ & \\
\hline Succumbed to another cause & $1 \quad(1.5)$ & $40 \quad(3.9)$ & \\
\hline Unknown & 3 (4.6) & $30 \quad(2.9)$ & \\
\hline
\end{tabular}

Percentage of patients with resection with curative intent is shown in parentheses.

and without postoperative infectious complications (Table II). Patients with infectious complications had a significantly higher frequency of diabetes mellitus and urgent surgery. We also found no statistical difference in the tumor location, incidence of laparoscopic surgery, inflammatory bowel disease, stoma construction, curability and tumor stage between the two groups. Fourteen (21.5\%) and $159(15.6 \%)$ patients, with and without infectious complications, respectively, had a relapse of cancer. Twelve (18.5\%) and 122 patients (12\%), with and without infectious complications, respectively, succumbed to recurrence of the primary tumor. One (1.5\%) and 40 patients, respectively, succumbed to other causes such as secondary malignancy, cerebral and heart infarction, arrhythmia and accidents. Although there is no difference in survival between patients with and without infectious complications, we found statistical difference in cancer-specific survival between the two groups (Fig. 1). Specifically, in stage III of colorectal cancer, patients with infectious complications had a poorer 
Table III. Univariate and multivariate analysis of factors that affect survival in patients with colorectal cancer.

\begin{tabular}{|c|c|c|c|c|c|c|}
\hline & \multicolumn{3}{|c|}{ Univariate } & \multicolumn{3}{|c|}{ Multivariate } \\
\hline & Hazard ratio & $95 \%$ CI & P-value & Hazard ratio & $95 \% \mathrm{CI}$ & P-value \\
\hline Age, 1-year increment & 1.03 & $1.02-1.05$ & $<0.0001$ & 1.05 & $1.03-1.06$ & $<0.0001$ \\
\hline Gender (male) & 1.04 & $0.76-1.42$ & 0.8000 & - & - & - \\
\hline Urgent operation (Yes) & 2.84 & $0.70-11.5$ & 0.1400 & - & - & - \\
\hline Laparoscopic operation (Yes) & 0.40 & $0.10-1.62$ & 0.2000 & - & - & - \\
\hline Tumor location (rectum) & 1.42 & $1.04-1.92$ & 0.0300 & 1.51 & $1.04-2.00$ & 0.0100 \\
\hline Infectious complications (Yes) & 1.58 & $0.88-2.85$ & 0.1300 & - & - & - \\
\hline Curability (A) & 0.21 & $0.14-0.32$ & 0.0010 & 0.50 & $0.31-1.29$ & 0.0600 \\
\hline \multicolumn{7}{|l|}{ Stage (compared with Stage I) } \\
\hline II & 2.92 & $1.41-6.06$ & 0.0040 & 2.65 & $1.27-5.52$ & 0.0100 \\
\hline III & 8.08 & $4.09-16.0$ & $<0.0001$ & 7.78 & $3.92-15.4$ & $<0.0001$ \\
\hline IV & 20.20 & 9.19-44.4 & $<0.0001$ & 12.5 & 4.41-35.4 & $<0.0001$ \\
\hline
\end{tabular}

CI, confidence interval.
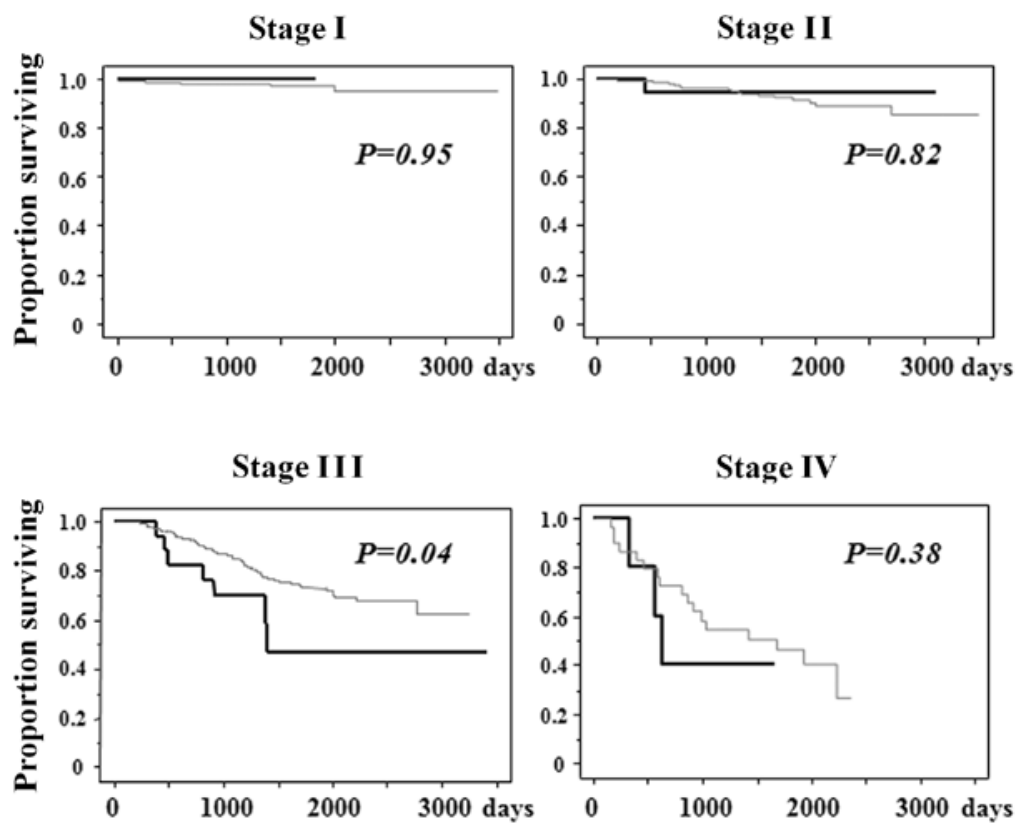

Figure 2. Overall survival after surgery with curative intent for colorectal cancer according to tumor stage. Bold and fine lines indicate the survival curve of patients with and without postoperative infectious complications, respectively. P-values of the log-rank test are shown in each graph.

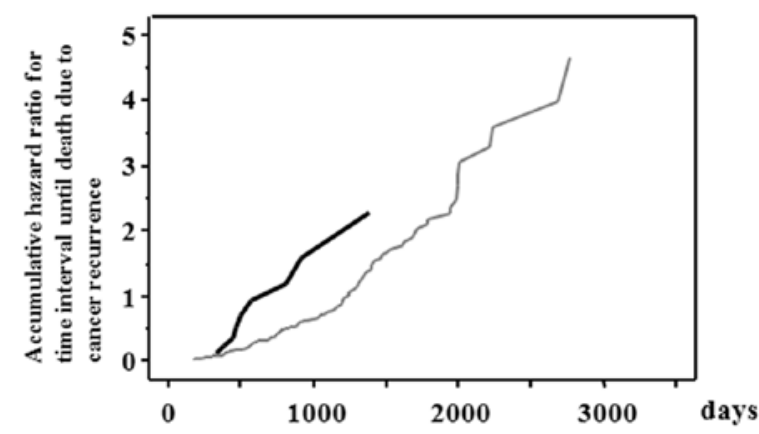

Figure 3. Accumulative hazard ratio for time interval until death due to cancer recurrence. Bold and fine lines indicate the survival curve of patients with and without postoperative infectious complications, respectively. cancer-specific survival than those without infectious complications (Fig. 2). This was not the case, however, with patients in stages I, II and IV of colorectal cancer.

In univariate analysis, age, tumor location, curability and tumor stage, but not postoperative infectious complications, were significantly associated with the overall survival rate (Table III). Multivariate analysis demonstrated that age, tumor location and tumor stage were significantly associated with overall survival.

Of the 134 patients who succumbed due to a recurrence of colorectal cancer, 52 (38.8\%), 95 (70.9\%) and 128 patients (95.5\%) had a recurrence of cancer before 12, 24 and 60 months, respectively. In order to identify which factors are 
Table IV. Univariate and multivariate analysis for time interval until patients succumbed due to recurrence from colorectal cancer.

\begin{tabular}{|c|c|c|c|c|c|c|}
\hline & \multicolumn{3}{|c|}{ Univariate analysis } & \multicolumn{3}{|c|}{ Multivariate analysis } \\
\hline & Hazard ratio & $95 \% \mathrm{CI}$ & P-value & Hazard ratio & $95 \% \mathrm{CI}$ & P-value \\
\hline Age, 1-year increment & 1.03 & $1.01-1.05$ & 0.001 & 1.01 & $0.99-1.03$ & 0.14 \\
\hline Gender (male) & 0.57 & $0.39-0.84$ & 0.005 & 0.60 & $0.41-0.88$ & 0.01 \\
\hline Emergency operation (Yes) & 2.00 & $0.28-14.5$ & 0.490 & - & - & - \\
\hline Laparoscopic operation (Yes) & 0.39 & $0.05-2.80$ & 0.350 & - & - & - \\
\hline Tumor location (rectum) & 1.27 & $0.87-1.84$ & 0.220 & - & - & - \\
\hline Infectious complications (Yes) & 2.10 & $1.09-4.07$ & 0.030 & 2.16 & $1.11-4.24$ & 0.03 \\
\hline Curability (A) & 0.78 & $0.48-1.25$ & 0.290 & - & - & - \\
\hline \multicolumn{7}{|l|}{ Stage (compared with stage I) } \\
\hline II & 1.01 & $0.34-2.94$ & 0.990 & - & - & - \\
\hline III & 1.14 & $0.42-3.13$ & 0.800 & - & - & - \\
\hline IV & 1.55 & $0.53-4.54$ & 0.430 & - & - & - \\
\hline
\end{tabular}

CI, confidence interval.

Table V. The influence of infectious complications for the time interval until patients succumbed due to cancer recurrence.

\begin{tabular}{lcccc}
\hline Infections & No. & Hazard ratio & $95 \%$ CI & P-value \\
\hline Anastomotic leakage & 29 & 3.48 & $1.49-8.14$ & 0.004 \\
Wound infection & 25 & 1.54 & $0.49-4.85$ & 0.470 \\
Urinary tract infection & 5 & 0.93 & $0.23-3.77$ & 0.920
\end{tabular}

CI, confidence interval.

Table VI. Main recurrence pattern of colorectal cancer.

\begin{tabular}{|c|c|c|c|c|c|c|c|}
\hline \multirow[t]{2}{*}{ Site of reccurence } & \multicolumn{3}{|c|}{ Complications } & \multicolumn{3}{|c|}{ No complications } & \multirow[t]{2}{*}{ P-value } \\
\hline & Total $(\%)$ & Colon & Rectum & Total (\%) & Colon & Rectum & \\
\hline Locoregional & $8(57.1)$ & 2 & 6 & $46(28.9)$ & 16 & 30 & \\
\hline Liver & $5(35.7)$ & 1 & 4 & $58(36.5)$ & 28 & 30 & \\
\hline Lung & $0 \quad(0.0)$ & 0 & 0 & $42(26.4)$ & 17 & 25 & 0.04 \\
\hline Distant lymph nodes & $0 \quad(0.0)$ & 0 & 0 & $11(6.9)$ & 2 & 9 & \\
\hline Brain & $0 \quad(0.0)$ & 0 & 0 & $1(0.6)$ & 0 & 1 & \\
\hline Bone & $1(7.1)$ & 1 & 0 & $1 \quad(0.6)$ & 0 & 1 & \\
\hline Total & $14(100.0)$ & 4 & 10 & $159(100.0)$ & 63 & 96 & \\
\hline
\end{tabular}

responsible for the time interval until patients succumbed to the disease due to cancer recurrence, univariate and multivariate analyses were performed (Table IV). Univariate analysis revealed that age, gender and infectious complications were associated with a shorter time interval of survival due to cancer recurrence, but this was not the case for tumor location, curability and tumor stage. Multivariate analysis revealed that infectious complications, as well as gender, were related with a shorter time interval of survival due to cancer recurrence. In patients with infectious complications, the mean time interval until death due to cancer recurrence was $708.8 \pm 114.4$ days, while that of patients without infectious complications was $1046.0 \pm 54.9$ days. The accumulative hazard ratio for time interval until death due to cancer recurrence was significantly higher in patients with infectious complications, than that in patients without infectious complications (Fig. 3). The 
univariate analysis performed with regard to the influence of infectious complications for the time interval for poor survival due to cancer recurrence showed that only an anastomotic leakage affects the time interval in patients with infectious complications (Table V). Patients with infectious complications had a more frequent locoregional relapse as compared to those without infectious complications as a main pattern of recurrence (Table VI).

\section{Discussion}

We demonstrated that complications due to postoperative infection are important prognostic factors after resection with curative intent for colorectal cancer, especially in patients with stage III disease. In addition, infectious complications were associated with time interval until patients succumbed due to the recurrence of colorectal cancer after resection for curative intent.

Although many previous reports demonstrated that postoperative infectious complications contribute to a high rate of recurrence and an unfavorable long-term survival in various malignancies $(5,7,8,13)$, the precise mechanism(s) relating longterm survival and postoperative infection remains unclear.

We also demonstrated that patients with infectious complications had more frequent locoregional relapse as compared to those without infectious complications. Substantial evidence exists indicating the presence of viable cancer cells in the bowel lumen of patients with colorectal cancer at the time of operation (14-16), which can be detected on suture or staple lines of anastomosis (17). Anastomotic leakage, the most frequent infectious complication found in this study, may lead to extraluminal implantation, which has the effect of upstaging the disease and increasing the incidence of locoregional relapse (13). Fujita et al demonstrated that the incidence of local recurrence in patients with anastomotic leakage was significantly higher than that in patients without leakage in colorectal cancer (18), which is consistent with our findings.

Another possible mechanism relating diminished survival and postoperative infection is a deregulated host immune response during infection that may contribute to tumorigenesis. It is known that inflammation caused by bacterial infection could develop systemic inflammatory response syndrome and lead to a shift towards a Th2-type lymphocyte pattern $(19,20)$, which is especially enhanced after surgical trauma (21-23). Th- 2 cytokines, such as IL-10, were shown to down-regulate tumor-specific immune responses by directly suppressing IFN $\gamma$ and IL-12 production. This caused a reduction in MHC expression on the surface of tumor cells and inhibited tumor antigen presentation by antigen-presenting cells (24-26). Taken together, these findings suggest that development of a postoperative Th-2 response during infectious complications following major surgical trauma likely contributes to the proliferation of occult or dormant cancer cells, resulting in decreased survival (27).

In conclusion, our study indicates that complications due to postoperative infection are a favorable predictor of adverse clinical outcome in patients with colorectal cancer. Further immunological study, however, is essential to substantiate our current data and to provide an assessment of their overall bio- logical effects. Nonetheless, more effort is required to prevent such postoperative infections to improve long-term as well as short-term survival in colorectal cancer patients.

\section{References}

1. Collins TC, Daley J, Henderson WH, et al: Risk factors for prolonged length of stay after major elective surgery. Ann Surg 230: 251-259, 1999.

2. Tsutsui S, Moriguchi S, Morita M, et al: Multivariate analysis of postoperative complications after esophageal resection. Ann Thorac Surg 53: 1052-1056, 1992.

3. Nowacki MP and Szymendera JJ: The strongest prognostic factors in colorectal carcinoma. Surgicopathologic stage of disease and postoperative fever. Dis Colon Rectum 26: 263-268, 1983.

4. Hirai $\mathrm{T}$, Yamashita $\mathrm{Y}$, Mukaida $\mathrm{H}$, et al: Poor prognosis in esophageal cancer patients with postoperative complications. Surg Today 28: 576-579, 1998.

5. Lagarde SM, De Boer JD, Ten Kate FJ, et al: Postoperative complications after esophagectomy for adenocarcinoma of the esophagus are related to timing of death due to recurrence. Ann Surg 247: 71-76, 2008.

6. Fucini C, Bandettini L, D'Elia M, et al: Are postoperative fever and/or septic complications prognostic factors in colorectal cancer resected for cure? Dis Colon Rectum 28: 94-95, 1985.

7. Akyol AM, McGregor JR, Galloway DJ, et al: Anastomotic leaks in colorectal cancer surgery: a risk factor for recurrence? Int $\mathbf{J}$ Colorectal Dis 6: 179-183, 1991.

8. Nespoli A, Gianotti L, Totis M, et al: Correlation between postoperative infections and long-term survival after colorectal resection for cancer. Tumori 90: 485-490, 2004.

9. Dionigi R, Dominioni L and Campani M: Infections in cancer patients. Surg Clin North Am 60: 145-159, 1980.

10. Wigmore SJ, McMahon AJ, Sturgeon CM, et al: Acute-phase protein response, survival and tumour recurrence in patients with colorectal cancer. Br J Surg 88: 255-260, 2001.

11. Varty PP, Linehan IP and Boulos PB: Intra-abdominal sepsis and survival after surgery for colorectal cancer. Br J Surg 81: 915-918, 1994.

12. Sobin LH and Fleming ID: TNM Classification of Malignant Tumors. 5th edition (1997). Union Internationale Contre le Cancer and the American Joint Committee on Cancer. Cancer 80: 1803-1804, 1997.

13. Walker KG, Bell SW, Rickard MJ, et al: Anastomotic leakage is predictive of diminished survival after potentially curative resection for colorectal cancer. Ann Surg 240: 255-259, 2004.

14. Keynes WM: Implantation from the bowel lumen in cancer of the large intestine. Ann Surg 153: 357-364, 1961.

15. Fermor B, Umpleby HC, Lever JV, et al: Proliferative and metastatic potential of exfoliated colorectal cancer cells. J Natl Cancer Inst 76: 347-349, 1986.

16. Skipper D, Cooper AJ, Marston JE, et al: Exfoliated cells and in vitro growth in colorectal cancer. Br J Surg 74: 1049-1052, 1987.

17. Gertsch P, Baer HU, Kraft R, et al: Malignant cells are collected on circular staplers. Dis Colon Rectum 35: 238-241, 1992.

18. Fujita S, Teramoto T, Watanabe M, et al: Anastomotic leakage after colorectal cancer surgery: a risk factor for recurrence and poor prognosis. Jpn J Clin Oncol 23: 299-302, 1993.

19. Ramer-Quinn DS, Baker RA and Sanders VM: Activated $\mathrm{T}$ helper 1 and $\mathrm{T}$ helper 2 cells differentially express the beta-2adrenergic receptor: a mechanism for selective modulation of $\mathrm{T}$ helper 1 cell cytokine production. J Immunol 159: 4857-4867, 1997.

20. Spolarics Z, Siddiqi M, Siegel JH, et al: Depressed interleukin12 -producing activity by monocytes correlates with adverse clinical course and a shift toward Th2-type lymphocyte pattern in severely injured male trauma patients. Crit Care Med 31: 1722-1729, 2003.

21. Aosasa S, Ono S, Mochizuki H, et al: Activation of monocytes and endothelial cells depends on the severity of surgical stress. World J Surg 24: 10-16, 2000.

22. Mokart D, Capo C, Blache JL, et al: Early postoperative compensatory anti-inflammatory response syndrome is associated with septic complications after major surgical trauma in patients with cancer. Br J Surg 89: 1450-1456, 2002. 
23. Tsujimoto H, Ono S, Majima $\mathrm{T}$, et al: Differential toll-like receptor expression after ex vivo lipopolysaccharide exposure in patients with sepsis and following surgical stress. Clin Immunol 119: 180-187, 2006.

24. Clerici M, Shearer GM and Clerici E: Cytokine dysregulation in invasive cervical carcinoma and other human neoplasias: time to consider the TH1/TH2 paradigm. J Natl Cancer Inst 90: 261-263, 1998.

25. Moore KW, O'Garra A, De Waal Malefyt R, et al: Interleukin-10. Annu Rev Immunol 11: 165-190, 1993.
26. Beissert S, Hosoi J, Grabbe S, et al: IL-10 inhibits tumor antigen presentation by epidermal antigen-presenting cells. J Immunol 154: 1280-1286, 1995.

27. Mynster T, Christensen IJ, Moesgaard F, et al: Effects of the combination of blood transfusion and postoperative infectious complications on prognosis after surgery for colorectal cancer Danish RANX05 Colorectal Cancer Study Group. Br J Surg 87: 1553-1562, 2000. 